\title{
Association of Relatively Short Posterior Mitral Leaflet With Mitral Regurgitation in Patients With Atrial Fibrillation
}

\author{
Hiroki Takada, MD; Hidekazu Tanaka, MD, PhD; Shun Yokota, MD; Jun Mukai, MD; \\ Makiko Suto, MD, PhD; Fumitaka Soga, MD, PhD; Yutaka Hatani, MD, PhD; \\ Hiroki Matsuzoe, MD, PhD; Keiko Hatazawa, MD, PhD; Kensuke Matsumoto, MD, PhD; \\ Koji Fukuzawa, MD, PhD; Ken-ichi Hirata, MD, PhD
}

\begin{abstract}
Background: The underlying mechanism of mitral regurgitation (MR) in atrial fibrillation (AF) is an isolated annulus dilation caused by left atrial (LA) remodeling. However, the association of mitral valve (MV) geometry with MR in AF patients remains unclear.

Methods and Results: We studied 96 AF patients with preserved left ventricular ejection fraction (LVEF). MV geometry was evaluated with 3-dimensional transesophageal echocardiography (3D-TEE). Mitral annulus area of the MR group ( $n=11, \geq$ moderate) was significantly larger (10.6 \pm 1.8 vs. $\left.8.2 \pm 1.5 \mathrm{~cm}^{2}, P<0.0001\right)$, and relative posterior mitral leaflet $(P M L)$ area ( $P M L$ area / mitral annulus area) was significantly smaller ( $0.51 \pm 0.06$ vs. $0.57 \pm 0.01, P=0.002)$ than in the non-MR group ( $n=85,<$ moderate). Multivariate logistic regression analysis showed that, in addition to LA volume index (LAVI), a relative PML area was independently associated with MR. For sequential logistic regression models to determine the association of MR, clinical variables including age, gender and LVEF were improved by the addition of LAVI $(P<0.001)$ and was improved by addition of mitral annulus area $(P=0.01)$, and further improved by addition of relative $P M L$ area $(P<0.001)$.
\end{abstract}

Conclusions: A relatively short PML plays an important role in the development of MR in AF patients. Assessment of MV geometry by 3D-TEE may thus have clinical implications for better surgical management of AF patients with significant MR.

Key Words: Atrial fibrillation; Echocardiography; Mitral regurgitation; Mitral valve

A trial fibrillation (AF) is the most common arrhythmia and is associated with several important cardiovascular events, including impaired quality of life, embolism events, heart failure (HF), and death. ${ }^{1-6} \mathrm{AF}$ and $\mathrm{HF}$ are frequently observed to coexist in clinical practice. AF causes left atrial (LA) remodeling and enlargement, with the latter possibly resulting in mitral annular dilatation, which in turn may cause progressive mitral regurgitation (MR), a potential mechanism by which AF induces $\mathrm{HF}$ despite the absence of left ventricular (LV) remodeling. Thus, the underlying mechanism of MR in AF is thought to be isolated annulus dilatation by LA remodeling, known as Carpentier's functional classification Type I. ${ }^{7}$ Mitral leaflets are able to adapt by means of some compensatory enlargement in response to mechanical stretching resulting from mitral annular dilatation caused by LA remodeling in AF patients. On the other hand, AF patients with significant MR are often seen in clinical practice to have a posterior mitral leaflet (PML) that is shortened in comparison with the anterior mitral leaflet (AML) (Figure 1).
It has been recently reported that the development of MR in AF patients is associated with not only mitral annular dilatation caused by LA enlargement, but also with a multiplicity of other factors. ${ }^{\mathbf{8}-12}$ However, the association of mitral valve (MV) geometry, especially in terms of the shortened PML, with MR in AF patients remains unclear. The purpose of our study was, therefore, to test the hypothesis that a shortened PML is associated with the development of Carpentier's functional classification Type I MR in AF patients, especially in those without LV remodeling.

\section{Methods}

\section{Study Population}

This study was a retrospective analysis of 96 AF patients who were referred for pulmonary vein isolation or cardioversion at Kobe University Hospital between February 2016 and September 2017. Patients were excluded from enrolment in the study if they met any of the following

Received June 11, 2019; revised manuscript received July 26, 2019; accepted August 13, 2019; J-STAGE Advance Publication released online September 13, 2019 Time for primary review: 28 days

Division of Cardiovascular Medicine, Department of Internal Medicine, Kobe University Graduate School of Medicine, Kobe, Japan

Mailing address: Hidekazu Tanaka, MD, PhD, FAHA, FACC, FASE, FJCS, Division of Cardiovascular Medicine, Department of Internal Medicine, Kobe University Graduate School of Medicine, 7-5-2 Kusunoki-cho, Chuo-ku, Kobe 650-0017, Japan. E-mail:tanakah@med.kobe-u.ac.jp

ISSN-1346-9843 All rights are reserved to the Japanese Circulation Society. For permissions, please e-mail: cj@j-circ.or.jp 


\section{Sinus rhythm without MR}

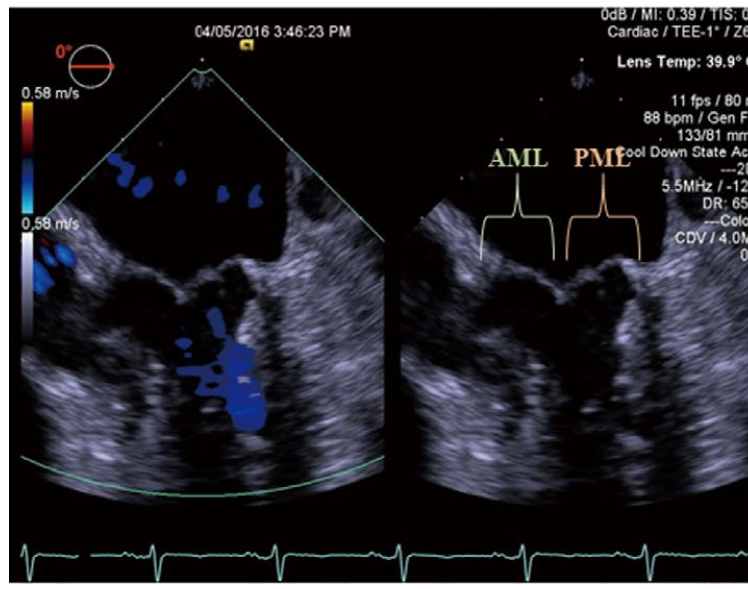

\section{AF with MR}

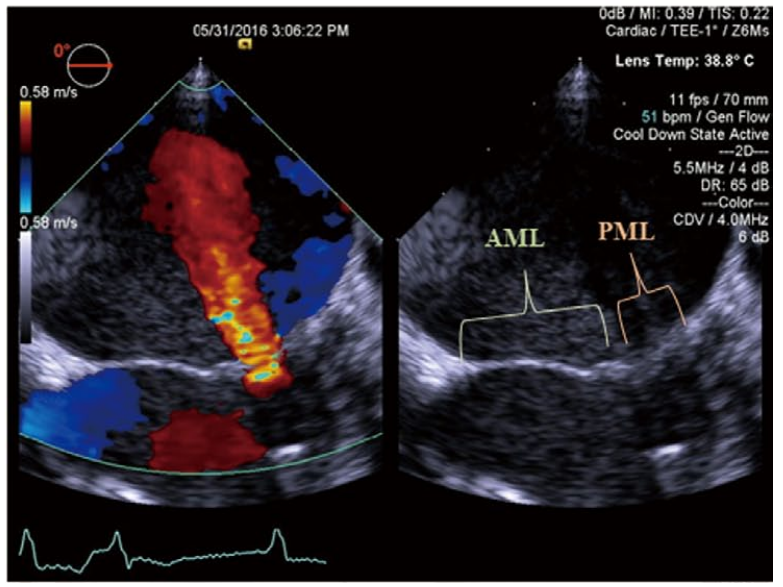

Figure 1. Representative cases of a patient with sinus rhythm without mitral regurgitation (MR) and an atrial fibrillation (AF) patient with significant MR, showing that AF patients with significant MR are more frequently observed with a posterior mitral leaflet (PML) that is shorter than the anterior mitral leaflet (AML).

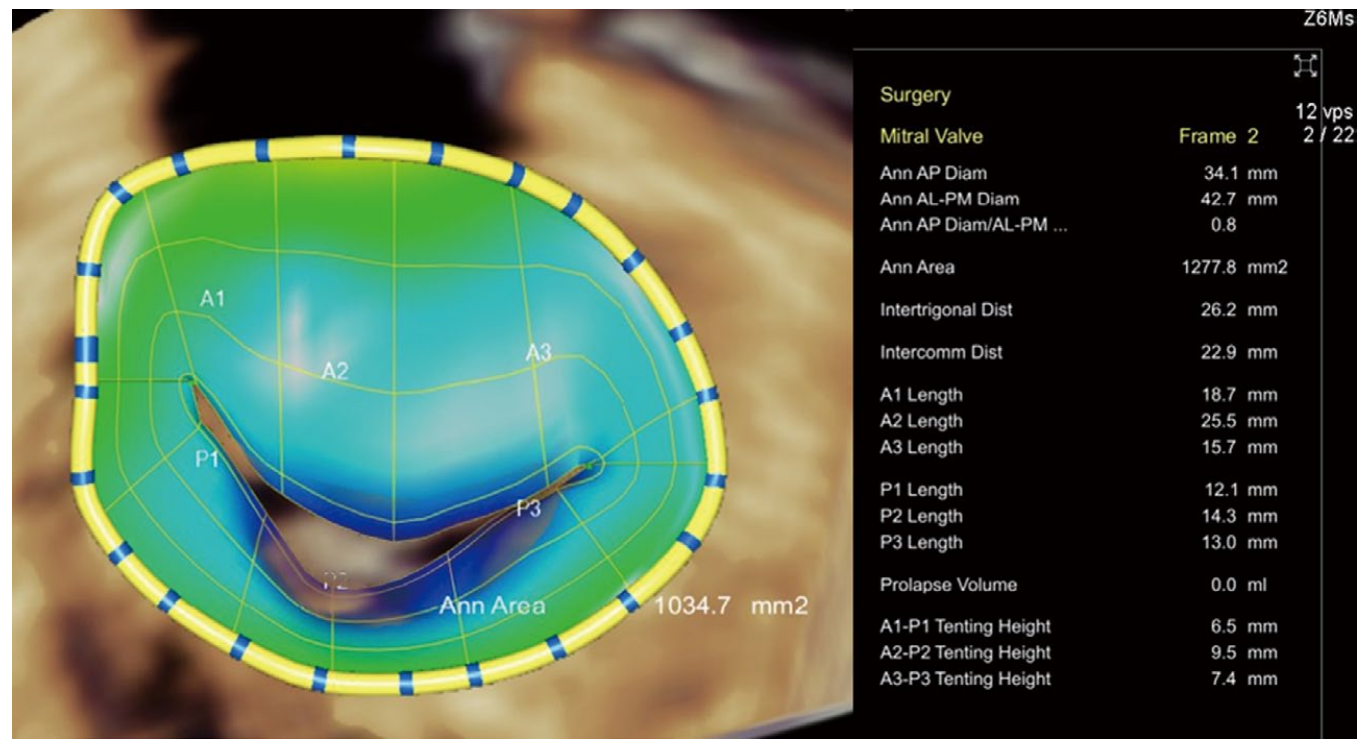

Figure 2. Assessment of mitral valve geometric parameters by 3D transesophageal echocardiography. Mitral annulus area, anterior $(\mathrm{AML})$ area and posterior mitral leaflet $(\mathrm{PML})$ area were determined in this study.

criteria: (1) LV ejection fraction (LVEF) $<50 \%$; (2) previous history of open-heart surgery; (3) serious renal dysfunction defined as glomerular filtration rate $<30 \mathrm{~mL} / \mathrm{min} / 1.73 \mathrm{~m}^{2}$; (4) uncontrolled hypertension $>180 / 100 \mathrm{mmHg}$; (5) more than moderate aortic valve disease; and (6) major cause of MR other than Carpentier's functional classification Type I. We also excluded patients with Carpentier's functional classification Type I MR who had PML hamstringing, in order to exclude the effect on the development of MR of the tip of the PML tethering towards the LV posterior wall.
This study was approved by the local institutional ethics committee (No. 180112).

\section{Echocardiographic Examination}

All transesophageal echocardiography (TEE) studies were performed with a commercially available echocardiographic system (ACUSON SC2000; Siemens Medical Solutions, Mountain View, CA, USA). Transthoracic echocardiography (TTE) studies were performed with other commercially available echocardiographic systems within 5 days of 
Table 1. Baseline Clinical and Echocardiographic Characteristics

\begin{tabular}{|c|c|c|c|c|}
\hline & $\begin{array}{l}\text { All patients } \\
(n=96)\end{array}$ & $\begin{array}{l}\text { Patients with MR } \\
(n=11)\end{array}$ & $\begin{array}{l}\text { Patients without MR } \\
\qquad(\mathrm{n}=85)\end{array}$ & $P$ value \\
\hline \multicolumn{5}{|l|}{ Clinical characteristics } \\
\hline Age (years) & $65.9 \pm 9.9$ & $70.7 \pm 10.8$ & $65.6 \pm 9.9$ & 0.12 \\
\hline Gender (female) [n (\%)] & $34(35.4)$ & $6(54.5)$ & $28(32.9)$ & 0.142 \\
\hline Body surface area $\left(\mathrm{m}^{2}\right)$ & $1.72 \pm 0.20$ & $1.49 \pm 0.21$ & $1.74 \pm 0.19$ & 0.001 \\
\hline Chronic AF [n (\%)] & $47(49.0)$ & $11(100)$ & $36(42.4)$ & $<0.001$ \\
\hline Paroxysmal AF [n (\%)] & $49(51.0)$ & $0(0)$ & $49(57.6)$ & $<0.001$ \\
\hline $\mathrm{CHADS}_{2}$ score & $1.35 \pm 1.31$ & $2.18 \pm 1.60$ & $1.26 \pm 1.23$ & 0.041 \\
\hline Congestive heart failure $[\mathrm{n}(\%)]$ & $16(16.7)$ & $8(72.7)$ & $8(9.4)$ & $<0.001$ \\
\hline Hypertension [n (\%)] & $51(53.1)$ & $6(54.5)$ & $45(52.9)$ & 0.92 \\
\hline Age (>75 years) $[n(\%)]$ & $20(20.8)$ & $5(45.5)$ & $15(17.6)$ & 0.048 \\
\hline Diabetes mellitus [n (\%)] & $20(20.8)$ & $1(9.1)$ & $19(22.4)$ & 0.28 \\
\hline Stroke / TIA [n (\%)] & $13(13.5)$ & $2(18.2)$ & $11(12.9)$ & 0.46 \\
\hline \multicolumn{5}{|l|}{ Echocardiographic parameters } \\
\hline LV end-diastolic diameter (mm) & $46.7 \pm 5.8$ & $51.4 \pm 7.9$ & $46.0 \pm 5.2$ & 0.004 \\
\hline LV end-systolic diameter (mm) & $30.5 \pm 5.6$ & $37.2 \pm 7.6$ & $29.6 \pm 4.7$ & 0.008 \\
\hline $\operatorname{LVEF}(\%)$ & $62.8 \pm 6.2$ & $64.7 \pm 6.8$ & $62.7 \pm 5.7$ & 0.704 \\
\hline Interventricular septum thickness (mm) & $10.2 \pm 2.3$ & $9.2 \pm 2.6$ & $10.3 \pm 2.2$ & 0.138 \\
\hline Posterior wall thickness $(\mathrm{mm})$ & $10.0 \pm 1.9$ & $9.6 \pm 1.5$ & $10.1 \pm 1.9$ & 0.593 \\
\hline $\mathrm{LAVI}\left(\mathrm{mL} / \mathrm{m}^{2}\right)$ & $50.7 \pm 30.0$ & $110.4 \pm 57.5$ & $43.3 \pm 16.8$ & $<0.0001$ \\
\hline $\mathrm{E}(\mathrm{cm} / \mathrm{s})$ & $79.4 \pm 24.6$ & $112.2 \pm 46.4$ & $73.8 \pm 17.3$ & 0.008 \\
\hline \multicolumn{5}{|l|}{ 3D mitral valve geometric parameters } \\
\hline $\mathrm{AML}$ area $\left(\mathrm{cm}^{2}\right)$ & $4.6 \pm 1.0$ & $5.8 \pm 1.1$ & $4.4 \pm 0.9$ & $<0.001$ \\
\hline $\mathrm{PML}$ area $\left(\mathrm{cm}^{2}\right)$ & $4.8 \pm 1.0$ & $5.3 \pm 1.0$ & $4.6 \pm 0.9$ & 0.041 \\
\hline Relative $\mathrm{AML}$ area & $0.54 \pm 0.07$ & $0.56 \pm 0.08$ & $0.54 \pm 0.07$ & 0.654 \\
\hline Relative PML area & $0.56 \pm 0.09$ & $0.51 \pm 0.06$ & $0.57 \pm 0.01$ & 0.002 \\
\hline $\mathrm{PML}$ area / AML area & $1.06 \pm 0.20$ & $0.93 \pm 0.19$ & $1.08 \pm 0.20$ & 0.021 \\
\hline Mitral annulus area $\left(\mathrm{cm}^{2}\right)$ & $8.48 \pm 1.74$ & $10.6 \pm 1.8$ & $8.2 \pm 1.5$ & $<0.0001$ \\
\hline A1 length $(\mathrm{mm})$ & $1.34 \pm 0.16$ & $1.47 \pm 0.13$ & $1.32 \pm 0.16$ & 0.003 \\
\hline A2 length (mm) & $1.91 \pm 0.25$ & $2.13 \pm 0.25$ & $1.88 \pm 0.24$ & 0.005 \\
\hline A3 length (mm) & $1.26 \pm 0.21$ & $1.5 \pm 0.37$ & $1.22 \pm 0.16$ & 0.003 \\
\hline P1 length (mm) & $1.21 \pm 0.16$ & $1.11 \pm 0.14$ & $1.22 \pm 0.16$ & 0.03 \\
\hline P2 length (mm) & $1.24 \pm 0.22$ & $1.08 \pm 0.25$ & $1.26 \pm 0.21$ & 0.032 \\
\hline P3 length (mm) & $1.29 \pm 0.21$ & $1.16 \pm 0.19$ & $1.3 \pm 0.21$ & 0.042 \\
\hline
\end{tabular}

Data are presented as mean $\pm \mathrm{SD}$, or $\mathrm{n}(\%)$. 3D, 3-dimensional; $\mathrm{AF}$, atrial fibrillation; $\mathrm{AML}$, anterior mitral leaflet; $\mathrm{E}$, peak early diastolic mitral flow velocity; EF, ejection fraction; LAVI, left atrial volume index; LV, left ventricular; PML; posterior mitral leaflet; TIA, transient ischemic attack.

the TEE studies (Xario SSA-660A; Aplio XG, and ARTIDA; Canon Medical Systems, Tochigi, Japan, or iE 33; Philips Medical Systems, Andover, MA, USA). Digital routine grayscale 2-D cine loops and tissue Doppler cine loops were obtained from 3 consecutive beats with endexpiratory apnea from standard apical and parasternal views. Sector width was optimized to allow for complete myocardial visualization while the frame rate was maximized regardless of heart rate. Standard TTE measurements were obtained in accordance with the current guidelines of the European Association of Cardiovascular Imaging. ${ }^{13}$

\section{Assessment of 3D MV Geometric Analysis}

3D MV geometric analysis was performed offline using semi-automated valve software (eSie Valves; Siemens Medical Solutions) as previously described in detail.14,15 Briefly, 3D digital imaging and communications in medicine data are loaded first, and a mid-systolic frame is chosen for subsequent analysis. The MV is then segmented automatically using a machine-learning algorithm. The position of the MV and its orientation and dimensions in the image are detected first to form a region of interest, within which key landmarks such as trigones and commissures as well as the annulus and leaflet free edges can be detected, after which an average surface model of the anterior and posterior leaflets is fitted to the landmarks. The shapes of these contours are then altered to match the atrial side of the leaflets in the image. To facilitate automated computation of complex measurements, the surface model returned by eSie Valves $^{\mathrm{TM}}$ (Siemens Healthineers, Erlangen, Germany) is represented uniformly. This is established from a landmarkbased resampling procedure. Each vertex of the valve surface model is uniquely defined by 2 coordinates: the $\mathrm{u}$-coordinate, tangential to the valve circumference, from anterior to posterior, and the v-coordinate, perpendicular to the valve circumference, from annulus to free edge. Once the MV is modeled, different workflow options are available to confirm the predetermined landmarks and edit the segmented valve (Figure 2). For this study, the mitral annulus area, AML area and PML area were measured, 


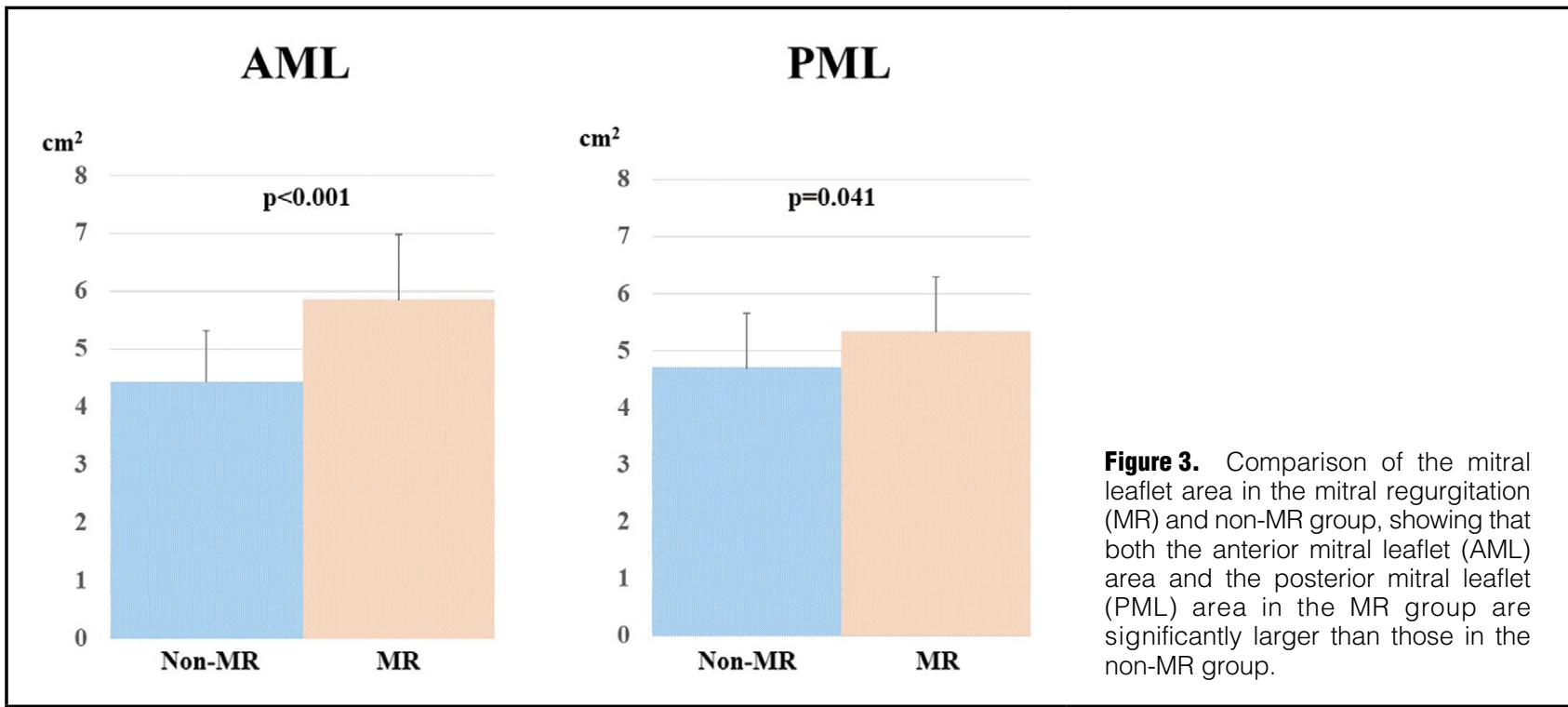

\section{Relative AML}

\section{Relative PML}

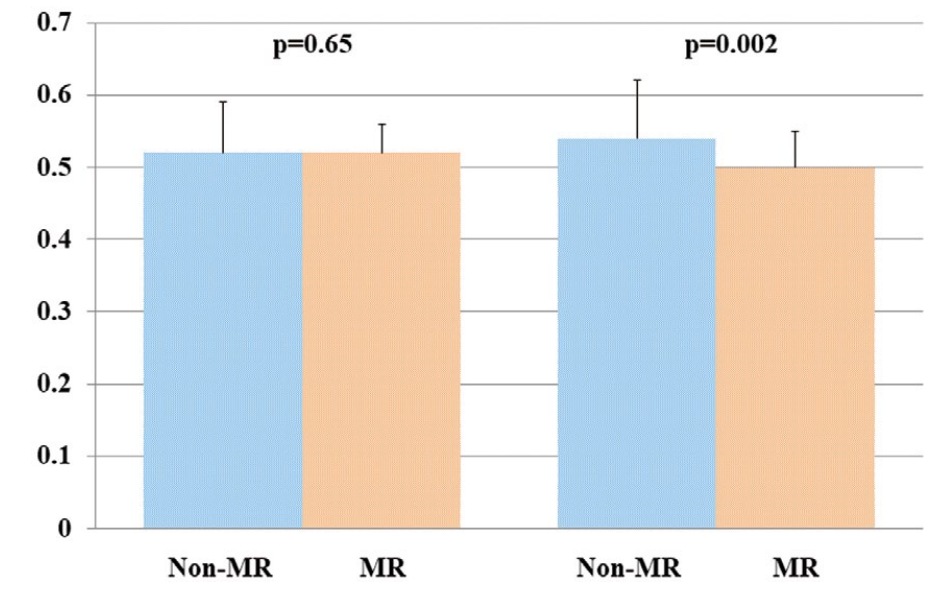

Figure 4. Comparison of the mitral leaflet area in the mitral regurgitation (MR) and non-MR groups, showing that the relative anterior mitral leaflet (AML) area of the MR group and the non-MR group is similar, but the relative posterior mitral leaflet (PML) of the MR group is significantly smaller than that of the non-MR group.

as well as the relative AML area (AML area / mitral annulus area) and relative PML area (PML area / mitral annulus area).

\section{Assessment of Severity of MR}

MR was quantified in the parasternal long-axis view of TTE by measuring the vena contracta width in the zoom mode at the narrowest portion of the MR jet as it emerges from the orifice. ${ }^{16}$ The severity of MR was graded as none or trace, mild $(<0.30 \mathrm{~cm})$, moderate $(0.30-0.69 \mathrm{~cm})$, or severe $(\geq 0.70 \mathrm{~cm})$. More than moderate MR was defined as significant.

\section{Statistical Analysis}

Continuous variables are expressed as mean values and standard deviation for normally distributed data, and as the median and interquartile range for non-normally distributed data. Categorical variables are expressed as frequencies and percentages. The parameters of subgroups were compared by Student's t-test or Mann-Whitney U test as appropriate, and proportional differences were evaluated by Fisher's exact test or the $\chi^{2}$ test as appropriate. The initial univariate regression analysis to identify univariate associated parameters of significant MR was followed by a multivariate regression model using enter selection. Sequential logistic models were constructed to determine the incremental benefit by using the relative PML area for determining the associations of significant MR over clinical variables including age, gender, LVEF, and LA volume index (LAVI) as well as the mitral annulus area. For all steps, a $\mathrm{P}$ value of $<0.05$ was regarded as statistically significant. The intraclass correlation coefficient was used to determine inter- and intra-observer reproducibility for 3D MV geometric parameters from 20 randomly selected subjects. All analyses were performed with commercially available software (SPSS software 
Table 2. Univariate and Multivariate Logistic Regression Analysis for the Association of Mitral Regurgitation

\begin{tabular}{|c|c|c|c|c|c|c|}
\hline \multirow{2}{*}{ Variables } & \multicolumn{3}{|c|}{ Univariate analysis } & \multicolumn{3}{|c|}{ Multivariate analysis } \\
\hline & OR & $95 \% \mathrm{Cl}$ & $P$ value & OR & $95 \% \mathrm{Cl}$ & $P$ value \\
\hline Age & 1.067 & $0.987-1.153$ & 0.105 & & & \\
\hline Gender & 2.443 & $0.686-8.699$ & 0.168 & & & \\
\hline LVEF & 0.969 & $0.872-1.076$ & 0.554 & & & \\
\hline LAVI & 1.141 & $1.057-1.232$ & 0.001 & 1.169 & $1.055-1.296$ & 0.003 \\
\hline Relative AML area (per 0.05 increment) & 1.068 & $0.834-1.367$ & 0.604 & & & \\
\hline Relative PML area (per 0.05 increment) & 0.538 & $0.362-0.800$ & 0.002 & 0.295 & $0.107-0.810$ & 0.018 \\
\hline $\mathrm{PML}$ area / AML area & 0.013 & $0.000-0.586$ & 0.026 & & & \\
\hline Mitral annulus area & 2.262 & $1.455-3.517$ & 0.001 & & & \\
\hline Hypertension & 1.067 & $0.302-3.764$ & 0.920 & & & \\
\hline Diabetes mellitus & 0.347 & $0.042-2.888$ & 0.328 & & & \\
\hline
\end{tabular}

$\mathrm{AML}$, anterior mitral leaflet; $\mathrm{Cl}$, confidence interval; OR, odds ratio; $\mathrm{PML}$, posterior mitral leaflet.

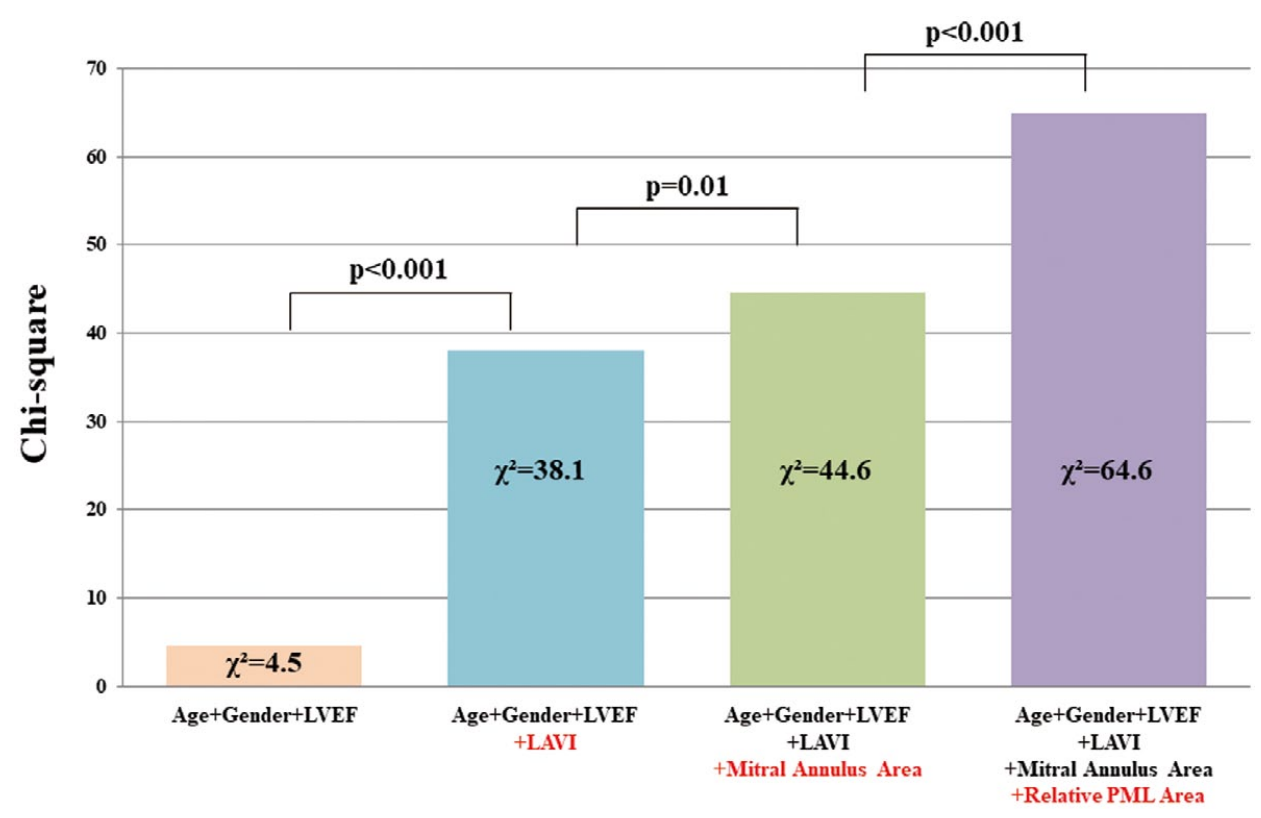

Figure 5. Sequential logistic regression models for the associations of mitral regurgitation (MR) show that the initial model based on clinical variables including age, gender and left ventricular ejection fraction (LVEF) was improved by the addition of the left atrial volume index (LAVI), then by the addition of the mitral annulus area, and further improved by the addition of the relative short posterior mitral leaflet (PML) area.

version 25.0, SPPS Inc., Chicago, IL, USA).

\section{Results}

\section{Patients' Characteristics}

The baseline clinical and TTE characteristics of the $96 \mathrm{AF}$ patients are summarized in Table 1. Their mean age was $66 \pm 10$ years, 34 patients $(35 \%)$ were female, and LVEF was $63 \pm 6 \%$. Chronic AF was diagnosed in 47 patients $(49 \%)$ and paroxysmal AF in the remaining 49 patients $(51 \%)$. Mean $\mathrm{CHADS}_{2}$ score was $1.4 \pm 1.3$.

\section{Comparison of Clinical and Echocardiographic Parameters of MR and Non-MR Groups}

The baseline clinical and TTE characteristics of patients in the MR and non-MR groups are summarized in Table 1. The MR group, comprising patients classified as having more than moderate MR, consisted of 11 patients $(11 \%)$, and the remaining 85 patients $(89 \%)$ made up the non-MR group. The 2 groups showed similar baseline clinical and echocardiographic characteristics, except for the fact that patients in the MR group were more likely to have a smaller body surface area $\left(1.49 \pm 0.21\right.$ vs. $1.74 \pm 0.19 \mathrm{~m}^{2}$, $\mathrm{P}=0.001)$, higher prevalence of chronic $\mathrm{AF}(100 \%$ vs. $42 \%$, $\mathrm{P}<0.001)$, higher $\mathrm{CHADS}_{2}$ score $(2.2 \pm 1.6$ vs. $1.3 \pm 1.2$, $\mathrm{P}=0.04$ ), larger $\mathrm{LV}$ dimensions ( $\mathrm{LV}$ end-diastolic diameter: $51.4 \pm 7.9$ vs. $46.0 \pm 5.2 \mathrm{~mm}, \mathrm{P}=0.004$ and $\mathrm{LV}$ end-systolic diameter: $37.2 \pm 7.6$ vs. $29.6 \pm 4.7 \mathrm{~mm}, \mathrm{P}=0.008$ ), larger LAVI $\left(110.4 \pm 57.5\right.$ vs. $\left.43.3 \pm 16.8 \mathrm{~mL} / \mathrm{m}^{2}, \mathrm{P}<0.0001\right)$, and a higher transmitral $\mathrm{E}$ wave $(112.2 \pm 46.4$ vs. $73.8 \pm 17.3 \mathrm{~cm} / \mathrm{s}, \mathrm{P}=0.008)$. 


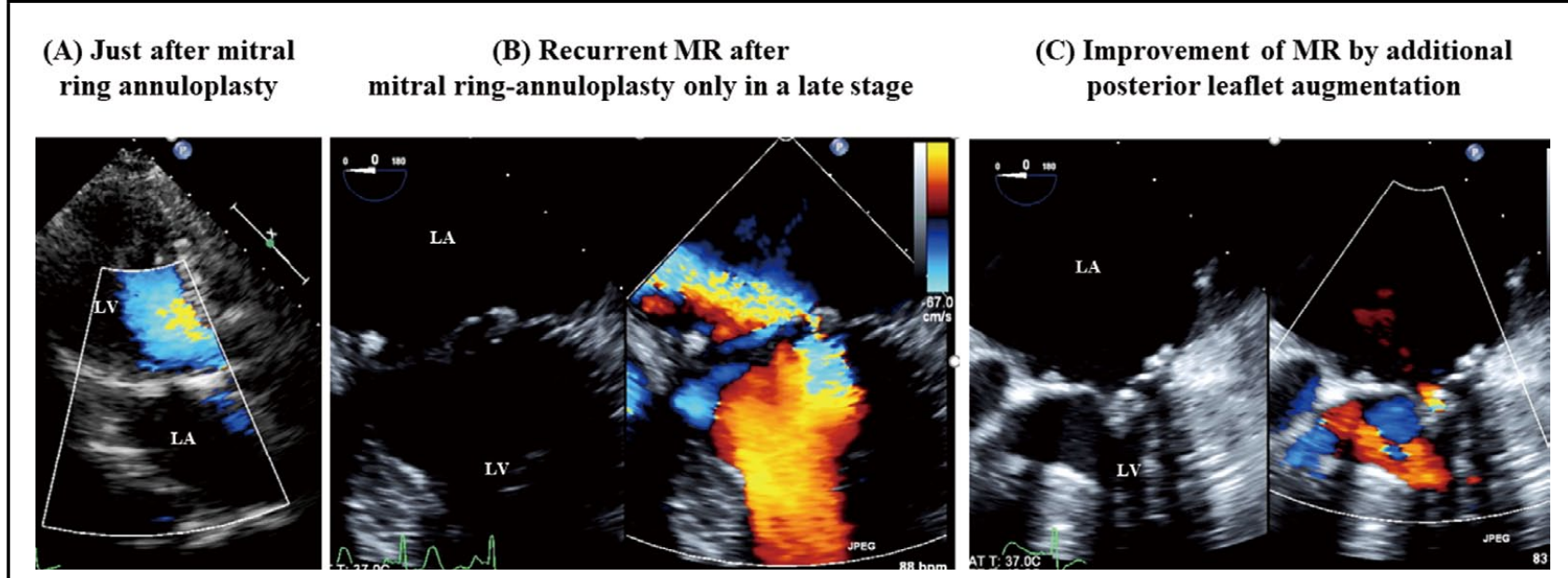

Figure 6. Patients with severe Carpentier Type I mitral regurgitation (MR) usually undergo mitral ring annuloplasty (A). However, atrial fibrillation (AF) patients with Carpentier Type I MR and a relatively short posterior mitral leaflet (PML) occasionally experience recurrent MR after mitral ring annuloplasty only, because of restricted PML motion in the late stage (B), and such patients may need additional procedures such as posterior mitral valve leaflet augmentation (C).

\section{Comparison of 3D MV Geometric Parameters of MR and Non-MR Groups}

As expected, the mitral annulus area of the MR group was significantly larger than that of the non-MR group $\left(10.6 \pm 1.8\right.$ vs. $\left.8.2 \pm 1.5 \mathrm{~cm}^{2}, \mathrm{P}<0.0001\right)$. In addition, the AML and PML areas of the MR group were significantly larger than those of the non-MR group (AML area: $5.8 \pm 1.1 \mathrm{vs.}$ $4.4 \pm 0.9 \mathrm{~cm}^{2}, \mathrm{P}<0.001$; PML area: $5.3 \pm 1.0$ vs. $4.6 \pm 0.9 \mathrm{~cm}^{2}$, $\mathrm{P}=0.04$; Figure 3). Interestingly, the relative $\mathrm{AML}$ area of the MR and non-MR groups was similar $(0.56 \pm 0.08$ vs. $0.54 \pm 0.07, \mathrm{P}=0.65$; Figure 4 Left), but the relative PML area of the MR group was significantly smaller than that of the non-MR group $(0.51 \pm 0.06$ vs. $0.57 \pm 0.01, \mathrm{P}=0.002$; Figure 4 Right). In addition, the lengths of P1, P2, and P3 of the MR group were significantly smaller than those of the non-MR group ( $\mathrm{P} 1: 1.11 \pm 0.14$ vs. $1.22 \pm 0.16 \mathrm{~cm} \mathrm{P}=0.03$, $\mathrm{P} 2: 1.08 \pm 0.25$ vs. $1.26 \pm 0.21 \mathrm{~cm} \mathrm{P}=0.032, \mathrm{P} 3: 1.16 \pm 0.19$ vs. $1.3 \pm 0.21 \mathrm{~cm} \mathrm{P}=0.042$ ). The lengths of $\mathrm{P} 1, \mathrm{P} 2$, and $\mathrm{P} 3$ also significantly correlated with relative $\mathrm{PML}$ area $(\mathrm{P} 1 \mathrm{r}=0.51$, $\mathrm{P}<0.0001, \mathrm{P} 2: \mathrm{r}=0.56, \mathrm{P}<0.0001, \mathrm{P} 3: \mathrm{r}=0.49, \mathrm{P}<0.0001)$.

\section{Associations of MR in AF Patients}

Univariate analysis using the logistic regression model showed that LAVI, relative PML area, PML area / AML area, and mitral annulus area were associated with MR. The odds ratio (OR) and $95 \%$ confidence interval (CI) for each of these variables are given in Table 2. An important finding of the multivariate logistic regression analysis was that the relative PML area (OR: $0.295 ; 95 \%$ CI $0.107-$ $0.810 ; \mathrm{P}=0.018$ ) proved to be an independently associated parameter of MR, as did LAVI.

The incremental advantage of using sequential logistic regression models for the association of MR is demonstrated in Figure 5. A model based on clinical variables including age, gender and LVEF $\left(\chi^{2}=4.5\right)$ was improved by the addition of the LAVI $\left(X^{2}=38.1 ; \mathrm{P}<0.001\right)$, then by the addition of the mitral annulus area $\left(\chi^{2}=44.6 ; \mathrm{P}=0.01\right)$, and further improved by the addition of the relative PML area $\left(\chi^{2}=64.6 ; \mathrm{P}<0.001\right)$.

\section{Reproducibility of 3D MV Geometric Parameters}

The intraclass correlation coefficients for interobserver reproducibility of 3D MV geometric parameters, including the mitral annulus the AML and the PML area, were 0.969 (95\% CI: 0.926-0.987), and 0.960 (95\% CI: 0.903-0.983), respectively, and the corresponding coefficients for intraobserver reproducibility were 0.969 (95\% CI: 0.924-0.987), and 0.967 (95\% CI: $0.920-0.986)$.

\section{Discussion}

The findings of this study indicated that a relatively short PML as assessed by 3D-TEE was independently associated with the development of MR in AF patients. Furthermore, in addition to LA dilatation and mitral annulus dilatation, a relatively short PML appears to be a valuable additional parameter in the development of MR in AF patients.

\section{MR in AF Patients}

AF can cause mitral annular dilation without LV dysfunction or dilation, which has been recognized by surgeons as the cause of Carpentier Type I MR. ${ }^{7}$ The mitral leaflets of $\mathrm{AF}$ patients can adapt by compensatory enlargement in response to mechanical stretching resulting from mitral annular dilatation caused by LA remodeling. However, if the mitral annulus dilates beyond the limits of MV leaflet adaptation, it impairs sufficient mitral leaflet co-adaptation and MR can occur as result. Moreover, long-lasting AF causes LA remodeling that also leads to morphological and functional changes in the MV apparatus. It has recently been reported that the development of MR in AF patients is associated with not only mitral annular dilatation caused by LA enlargement, but also with multiple other factors. ${ }^{8-12}$ Machino-Ohtsuka et al reported that, in addition to PML tethering and mitral annular dilatation, a variety of other factors, including a flattened mitral annular saddle shape, mitral annulus contractile dysfunction, and PML tethering, were associated with the development of MR in chronic AF patients and that PML tethering and mitral annular 
dilatation presented a higher standardized regression coefficient than the other variables. ${ }^{10} \mathrm{Kim}$ et al found that the AML and PML areas, as assessed by 3D-TEE in non-valvular chronic AF patients without LV dysfunction or remodeling, were significantly larger in response to mitral annular dilation compared with those in non-AF subjects, and that both the AML and PML areas in AF patients with MR were significantly larger than those in AF patients without MR. ${ }^{9}$ They also showed that, after the asymmetrical remodeling of the mitral annular geometry in the development of MR, the posterior-to-anterior mitral leaflet area ratio remained constant, whereas the posterior-to-anterior mitral annulus perimeter increased, while the posteriorto-anterior mitral annulus perimeter stayed unchanged. Finally, Kagiyama et al reported that in AF patients, insufficient mitral leaflet adaptation to annular dilatation, such as reflected in the ratio of total mitral leaflet area / mitral annulus area as assessed by means of 3D-TEE, rather than to mitral annular dilatation, was strongly associated with the severity of MR. ${ }^{11}$

It was recently reported that geometric change of the PML is associated with significant MR in AF patients without LV remodeling. Ito et al showed by means of 3D-TEE that, in addition to LA remodeling and mitral annular dilatation, the AML was flattened along the mitral annular plane, whereas the PML was bent towards the LV cavity, known as PML hamstringing, in AF patients with significant MR.12 This functional restriction of the PML has traditionally occurred because of the giant LA that is seen in advanced rheumatic MV disease, ${ }^{17}$ and AF patients are also likely to have PML hamstringing in association with severe LA dilatation. Because the purpose of this study was to investigate only the association of a relatively short PML with the development of MR in AF patients, we excluded AF patients with PML hamstringing, which may strongly affect the development of MR. In this study a relatively short PML was associated with the development of MR in AF patients, as well as mitral annulus dilatation. Moreover, PML area was larger but PML length, including P1, P2, and P3, was smaller in the MR group than in the non-MR group. Thus, the transverse extension of the PML caused by transverse dilatation of the mitral annulus rather than by longitudinal shortening of the PML may be associated with the development of $\mathrm{MR}$ in AF patients.

The precise mechanism of the occurrence of a relatively short PML in AF patients remains unclear. Several investigators have reported local immunologic inflammatory responses are associated with the LA in AF patients, so a relatively short PML in AF patients may be related to this. ${ }^{18-20}$ Other studies are required to provide pathological evidence for the mechanism of the occurrence of a relatively short PML in AF patients.

\section{Clinical Implications}

Symptomatic AF patients with severe Carpentier Type I MR usually undergo mitral ring annuloplasty (Figure 6A). However, AF patients with Carpentier Type I MR and a relatively short PML in the late stage occasionally experience recurrent $\mathrm{MR}$ after mitral ring annuloplasty only, because of the restricted PML motion (Figure 6B). Such patients may thus need an additional procedure such as posterior MV leaflet augmentation (Figure 6C). Therefore, the identification of a relatively short PML in AF patients by means of $3 \mathrm{D}-\mathrm{TEE}$ can be expected to be reliable, and may well have clinical implications for better surgical management of symptomatic AF patients with significant MR.

\section{Study Limitations}

This study covered a small number of patients in a singlecenter retrospective study, so future studies involving larger numbers of patients are required to verify our findings. Another limitation of this study was that the irregular rhythm of AF can affect the evaluation of MR severity. In addition, the assessment of MR severity in this study was performed by a semiquantitative method not quantitative method such as regurgitant volume, regurgitant fraction, or effective regurgitant orifice area. Finally, MR in patients with AF may change according to hemodynamics. However, all patients in this study were in a clinically stable condition at the time of TTE and TEE studies.

\section{Conclusions}

In addition to mitral annulus dilatation, a relatively short PML plays an important role in the development of MR in $\mathrm{AF}$ patients. The assessment of a relatively short PML by means of 3D-TEE is thus a valuable additive parameter for better surgical management of AF patients with significant MR.

\section{References}

1. Govindan M, Kiotsekoglou A, Saha SK, Camm AJ. Right atrial myocardial deformation by two-dimensional speckle tracking echocardiography predicts recurrence in paroxysmal atrial fibrillation. J Echocardiogr 2017; 15: 166-175.

2. Shin SH, Park MY, Oh WJ, Hong SJ, Pak HN, Song WH, et al. Left atrial volume is a predictor of atrial fibrillation recurrence after catheter ablation. J Am Soc Echocardiogr 2008; 21: $697-$ 702.

3. Boriani G, Proietti M. Atrial fibrillation prevention: An appraisal of current evidence. Heart 2018; 104: 882-887.

4. Wang TJ, Larson MG, Levy D, Vasan RS, Leip EP, Wolf PA, et al. Temporal relations of atrial fibrillation and congestive heart failure and their joint influence on mortality: The Framingham Heart Study. Circulation 2003; 107: 2920-2925.

5. Dorian P, Jung W, Newman D, Paquette M, Wood K, Ayers GM, et al. The impairment of health-related quality of life in patients with intermittent atrial fibrillation: Implications for the assessment of investigational therapy. J Am Coll Cardiol 2000; 36: $1303-1309$.

6. Mochizuki A, Yuda S, Fujito T, Kawamukai M, Muranaka A, Nagahara D, et al. Left atrial strain assessed by three-dimensional speckle tracking echocardiography predicts atrial fibrillation recurrence after catheter ablation in patients with paroxysmal atrial fibrillation. $J$ Echocardiogr 2017; 15: 79-87.

7. Carpentier A, Chauvaud S, Fabiani JN, Deloche A, Relland J, Lessana A, et al. Reconstructive surgery of mitral valve incompetence: Ten-year appraisal. J Thorac Cardiovasc Surg 1980; 79: $338-348$.

8. Ito K, Abe Y, Watanabe H, Shimada Y, Shibayama K, Oe H, et al. Prognostic significance of residual functional mitral regurgitation in hospitalized heart failure patients with chronic atrial fibrillation and preserved ejection fraction after medical therapies. $J$ Echocardiogr, doi:10.1007/s12574-018-0412-6.

9. Kim DH, Heo R, Handschumacher MD, Lee S, Choi YS, Kim KR, et al. Mitral valve adaptation to isolated annular dilation: Insights into the mechanism of atrial functional mitral regurgitation. JACC Cardiovasc Imaging 2019; 12: 665-677.

10. Machino-Ohtsuka T, Seo Y, Ishizu T, Sato K, Sugano A, Yamamoto $\mathrm{M}$, et al. Novel mechanistic insights into atrial functional mitral regurgitation: 3-dimensional echocardiographic study. Circ J 2016; 80: 2240-2248.

11. Kagiyama N, Hayashida A, Toki M, Fukuda S, Ohara M, Hirohata A, et al. Insufficient leaflet remodeling in patients with atrial fibrillation: Association with the severity of mitral regurgitation. Circ Cardiovasc Imaging 2017; 10: pii: e005451. 
12. Ito $\mathrm{K}$, Abe $\mathrm{Y}$, Takahashi $\mathrm{Y}$, Shimada $\mathrm{Y}$, Fukumoto $\mathrm{H}$, Matsumura $Y$, et al. Mechanism of atrial functional mitral regurgitation in patients with atrial fibrillation: A study using three-dimensional transesophageal echocardiography. $J$ Cardiol 2017; 70: $584-590$

13. Lang RM, Badano LP, Mor-Avi V, Afilalo J, Armstrong A, Ernande L, et al. Recommendations for cardiac chamber quantification by echocardiography in adults: An update from the American Society of Echocardiography and the European Association of Cardiovascular Imaging. Eur Heart J Cardiovasc Imaging 2015; 16: 233-270.

14. Ionasec RI, Voigt I, Georgescu B, Wang Y, Houle H, VegaHiguera F, et al. Patient-specific modeling and quantification of the aortic and mitral valves from 4-D cardiac CT and TEE. IEEE Trans Med Imaging 2010; 29: 1636-1651.

15. Calleja A, Poulin F, Woo A, Meineri M, Jedrzkiewicz S, Vannan MA, et al. quantitative modeling of the mitral valve by threedimensional transesophageal echocardiography in patients undergoing mitral valve repair: Correlation with intraoperative surgical technique. J Am Soc Echocardiogr 2015; 28: 1083-1092.

16. Zoghbi WA, Adams D, Bonow RO, Enriquez-Sarano M, Foster
E, Grayburn PA, et al. Recommendations for noninvasive evaluation of native valvular regurgitation: A report from the American Society of Echocardiography Developed in Collaboration with the Society for Cardiovascular Magnetic Resonance. $J$ Am Soc Echocardiogr 2017; 30: 303-371.

17. Beppu S, Kawazoe K, Nimura Y, Nagata S, Park YD, Sakakibara H, et al. Echocardiographic study of abnormal position and motion of the posterobasal wall of the left ventricle in cases of giant left atrium. Am J Cardiol 1982; 49: 467-472.

18. Suzuki A, Fukuzawa K, Yamashita T, Yoshida A, Sasaki N, Emoto T, et al. Circulating intermediate CD14++CD16+monocytes are increased in patients with atrial fibrillation and reflect the functional remodelling of the left atrium. Europace 2017; 19: $40-47$.

19. Yamashita T, Sekiguchi A, Iwasaki YK, Date T, Sagara K, Tanabe $\mathrm{H}$, et al. Recruitment of immune cells across atrial endocardium in human atrial fibrillation. Circ J 2010; 74: 262270.

20. Frustaci A, Chimenti C, Bellocci F, Morgante E, Russo MA, Maseri A. Histological substrate of atrial biopsies in patients with lone atrial fibrillation. Circulation 1997; 96: 1180-1184. 